abfall auf, der bei Methoxylierung oder Glucosidierung in 6-Stellung ausbleibt. Scopoletin und Äsculin sind sich verhältnismäßig ähnlich, jedoch sind zwei Unterschiede vorhanden, die auch bei den an Lösungen durchgeführten Messungen hervortraten ${ }^{2,6}: 1$. Im Bereich zwischen $p_{\mathrm{H}} 3$ und $p_{\mathrm{H}} 5,5$ wird die Fluoreszenzintensität von Äsculin relativ mehr geschwächt als die von Scopoletin. 2. Der Anstieg der Fluoreszenzintensität beim Ubergang nach höheren $p_{\mathrm{H}}$-Werten erfolgt bei Äsculin früher (unterhalb $p_{\mathrm{H}} 6$ ) als bei Scopoletin (oberhalb $p_{\mathrm{H}} 6$ ). Die abgebildeten Kurven stimmen weitgehend mit den an Lösungen gewonnenen überein ${ }^{2,6}$. Es muß jedoch allgemein beachtet werden, daß für einen strengen Vergleich derartiger Kurven auch eine gleiche Versuchsanordnung erforderlich ist, da Faktoren wie z. B. Art des Puffers, Fluoreszenzlöschung durch Verunreinigungen, spektrale Empfindlichkeit der Photozelle, Art des Sperrfilters und anderes die Meßergebnisse beeinflus$\operatorname{sen}^{7}$.

Herrn Professor Dr. A. Küнn, Max-Planck-Institut für Biologie, Tübingen, bin ich für die freundliche Erlaubnis zur Benutzung des Fluorometers zu Dank verpflichtet.

\section{Über den Einfluß von Kochsalz auf die Ribonuclease und ihre Hemmung durch Heparin}

\author{
Von Bruno Lorenz, Rita Lorenz und Nepomuk Zöllner \\ Medizinische Poliklinik der Universität München, \\ (Direktor: Prof. Dr. W. SeITz) \\ (Z. Naturforschg. 15 b, 62-63 [1960]; eingeg. am 26. Oktober 1959)
}

Heparin hemmt die Ribonuclease des Pankreas in einer qualitativ gut $\mathrm{zu}$ beschreibenden Weise ${ }^{\mathbf{1}}$; die Ribonuclease-Hemmung ist demgemäß zur Heparinbestimmung prinzipiell brauchbar. Die Anwendung entsprechender Methoden auf eiweißhaltige Versuchsansätze stößt jedoch auf Schwierigkeiten, da unter den Bedingungen der $\mathrm{Kunit} \mathrm{z}$ schen RibonucleaseMethode ${ }^{2}$ dabei Trübungen durch Nucleoprotein-Bildung auftreten, welche die Durchführung dieses optischen Tests verhindern. Diese Trübung kann durch $\mathrm{Zu}$ satz von Kochsalz hintangehalten werden, doch beeinflußt der Salzzusatz sowohl die Aktivität der Ribonuclease als auch ihre Hemmung durch Heparin.

Carter und Greenstein ${ }^{3}$ und seither eine Reihe von Autoren ${ }^{4-7}$ haben mit verschiedenen Methoden beobachtet, daß Salze die Ribonuclease aktivieren. Wir selbst haben mit einer Methode, bei der die Spaltprodukte vom Ansatz abgetrennt und ihre Extinktion gemessen wird ${ }^{8}$ durch 0,04-m. Kochsalzzusatz eine Aktivierung auf das Dreifache der Reaktionsgeschwindigkeit in salzfreier Lösung erreicht; die Wirkung einiger anderer Chloride, Sulfate und Phosphate von Alkalien und Erdalkalien war quantitativ ähnlich.

Die Durchtestung verschiedener NaCl-Konzentrationen mit der Kunitz-Methode ergab zunächst ein scheinbares Maximum der Aktivierung bei 0,05-m. $\mathrm{NaCl}, \mathrm{NaCl}-K o n z e n t r a t i o n e n ~ u ̈ b e r ~ 0,2-m$. schienen sogar hemmend zu wirken. Mit dem Kochsalzzusatz nimmt jedoch auch die Extinktion sowohl des Ribonucleates als auch der Spaltprodukte erschöpfender RibonucleaseEinwirkung ab, die Veränderungen der Lichtabsorption

1 N. Zöllner u. J. Fellig, Amer. J. Physiol. 173, 223 [1953]; Naturwissenschaften 39, 523 [1952].

2 M. Kunitz, J. biol. Chemistry 164, 563 [1946].

3 C. E. Carter u. J. P. Greenstein, J. nat. Cancer Inst. 7, 29 [1946/47].

4 C. Lamanna u. M. F. Mallette, Arch. Biochemistry 24, 451 [1949].

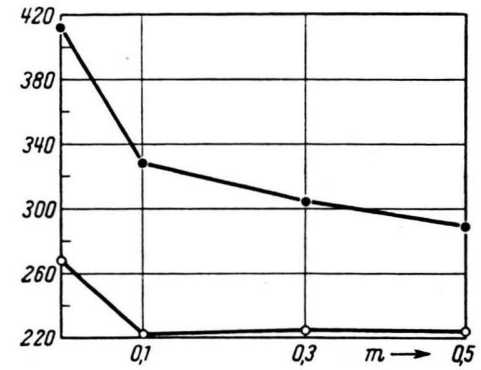

Abb. 1. Der Einfluß von Kochsalz auf die Lichtabsorption von Ribonucleat (obere Kurve) und ihres RibonucleaseAbbauproduktes (untere Kurve). Der Ansatz enthielt $2 \mathrm{mg}$ Ribonucleat und Kochsalz, um die angegebene Molarität zu erreichen, in $5 \mathrm{~cm}^{3}$ eines $0,04-m$. Natriumacetat-Puffers von $p_{\mathrm{H}}$ 5,0. Zum erschöpfenden Abbau wurden $5 \gamma$ Ribonuclease verwendet. Ordinate: Extinktion $\cdot 10^{3}$ (1-cm-Küvette) bei $300 \mathrm{~m} \mu$. Abszisse: NaCl-Konzentration.

laufen aber nicht parallel (Abb. 1). (Der Abfall der Extinktion des Ribonucleates bei steigender NaCl-Konzentration stimmt quantitativ überein mit Befunden von Shack, Jenkins und Thompsetr ${ }^{9}$, die an Desoxyribonucleinsäure aus Kalbsthymus ebenfalls einen Abfall der Lichtabsorption mit steigender Salzkonzentration fanden.) Die für die Berechnung der RibonucleaseAktivität wesentliche Differenz zwischen Extinktion der Ribonucleinsäure und ihrer Spaltprodukte $\left(E_{0}-E_{\mathrm{f}}\right)$ nimmt also im Bereich der geprüften Salzkonzentrationen laufend ab. Daraus folgt, daß zwar die erwähnte Beschleunigung des Extinktionsabfalles bei der Einwirkung von Ribonuclease auf Ribonucleinsäure durch Salze eine echte Aktivierung darstellt, daß aber in Versuchen, in denen der Extinktionsabfall bei Salzzusatz geringer als ohne diesen ist, geprüft werden muß, ob die Verringerung des Extinktionsabfalles Ausdruck einer Hemmung der Ribonuclease oder die Folge der Verkleinerung von $E_{0}-E_{\mathrm{f}}$ ist.

Die Berechnung der Aktivierung der Ribonuclease durch Salz ergibt unter Anwendung der K u n i t z schen

5 H. Edelhoch u. J. Coleman, J. biol. Chemistry 219, 351 [1956].

6 S. R. Dickman, R. B. Kropf u. J. P. Aroskar, Biochim. biophysica acta [Amsterdam] 21, 539 [1956].

7 S.R. Dickman u. B. Ring, J. biol. Chemistry 231, 741 [1958].

8 N. Zöllner, Habilitationsschrift, München 1954.

9 J. Shack, R. J. Jenkins u. J. M. Thompsett, J. biol. Chemistry 203, 373 [1953]. 
Formeln ${ }^{2}$ bei $0,05-m$. $\mathrm{NaCl}$ eine Aktivierung auf das 2,2-fache. Bei 0,5-m. NaCl betrug der Extinktionsabfall in den ersten 10 Min. 0,040, während unter sonst gleichen Bedingungen ohne Salzzusatz der Extinktionsabfall 0,067 ausmachte. Setzt man die Werte für $E_{0}-E_{\mathrm{f}}$ ein $(0,065$ für den Versuch mit $0,5-m$. $\mathrm{NaCl}, 0,171$ für den ohne), so ergibt sich dennoch eine Aktivierung

\begin{tabular}{|c|c|c|c|}
\hline $\begin{array}{c}\text { NaCl- } \\
\text { Konzentration }\end{array}$ & $\begin{array}{c}\text { Versuch mit } \\
\text { Heparinzusatz } \\
(50 \gamma)(\mathrm{H})\end{array}$ & $\begin{array}{c}\text { Kontroll- } \\
\text { versuch (K) }\end{array}$ & $\mathrm{H}: \mathbf{K}$ \\
\hline$[-m]$. & & & \\
0 & 0,001 & 0,032 & 0,03 \\
0,03 & 0,024 & 0,048 & 0,50 \\
0,05 & 0,035 & 0,052 & 0,67 \\
0,1 & $\mathbf{0 , 0 5 0}$ & 0,048 & $\mathbf{1 , 0 4}$ \\
\hline
\end{tabular}

Tab. 1. Die Wirkung von Kochsalz auf die Hemmung der Ribonuclease durch Heparin. Ansatz wie in Abb. 1. Die Zahlen in Spalte 2 und 3 geben den Abfall der Extinktion bei $300 \mathrm{~m} \mu$ zwischen $15 \mathrm{Sek}$. und $10 \mathrm{Min}$. nach Versuchsbeginn wieder. durch Salz; direkte Bestimmungen unter Messung der Reaktionsprodukte haben wir bei dieser Salzkonzentration aber nicht angestellt.

(Dickman und Ring ${ }^{7}$ haben ebenfalls beobachtet, daß nach Salzzusatz sowohl die Lichtabsorption $(300 \mathrm{~m} \mu)$ von Ribonucleat bei $p_{\mathrm{H}} 5,0$ abnimmt, als auch bei höheren Konzentrationen der Extinktionsabfall unter der Einwirkung von Ribonuclease geringer wird. Bei ihrer Deutung, daß Salz die photometrisch meßbare Reaktion hemmt, haben die Autoren jedoch nicht berücksichtigt, $\mathrm{da} \beta E_{0}-E_{\mathrm{f}}$ mit steigender Salzkonzentration ebenfalls kontinuierlich abnimmt.)

Untersucht man nun, wie sich die Hemmung der Ribonuclease durch Heparin bei verschiedenen $\mathrm{NaCl}$ Konzentrationen verhält, so ergibt sich, daß zunehmende $\mathrm{NaCl}-\mathrm{Konzentrationen} \mathrm{die} \mathrm{Hemmung} \mathrm{weitgehend}$ zurückdrängen (Tab. 1). NaCl-Konzentrationen, wie sie zur Hintanhaltung der eingangs erwähnten Nucleoprotein-Bildung nötig sind $(0,2-0,4-m$.), drängen die Heparinhemmung soweit zurück, daß in ihrer Anwesenheit die Bestimmung kleiner Heparinmengen mit Hilfe des optischen Ribonuclease-Tests von KunITz unmöglich ist.

\section{Über die Schutzwirkung einiger Salycil-Derivate bei akutem experimentellem Adrenalin- Lungenödem}

Von Pietro De Bonis und Giuseppe Lomeo

Institut Clinica Medica und Terapia Medica der Universität Rom (Direktor: Prof. Dr. Luigr Condorelui)

(Z. Naturforschg. 15 b, 63-65 [1960] ; eingeg. am 16. November 1959)

In unseren Untersuchungen zur Klärung des Wirkungsmechanismus antirheumatischer Medikamente fanden wir, daß einige Pyrazolon-Derivate wie Antipyrin, Pyramidon, Novalgin, Butazolidin, bei akutem experimentellem Adrenalin-Lungenödem im Kaninchen eine
Schutzwirkung ausüben ${ }^{1}$. Um unsere Versuche zu vervollständigen, haben wir versucht, unter den gleichen Bedingungen die Wirkung einiger Salicyl-Präparate zu ermitteln, da dieselben, trotz ihrer sehr verschiedenen chemischen Konstitution und pharmakologischen Eigenschaften, mit den obengenannten antirheumatischen Substanzen eine erhebliche therapeutische Wirkung auf dem Gebiet der rheumatischen Erkrankungen gemeinsam haben.

Die erhaltenen Resultate sind in Tab. 1 aufgeführt.

Die angeführten Ergebnisse beweisen deutlich, daß die von uns verwendeten Salicyl-Derivate sich wie die in den vorhergehenden Untersuchungen geprüften antirheumatischen Substanzen verhalten, indem sie unter geeigne-

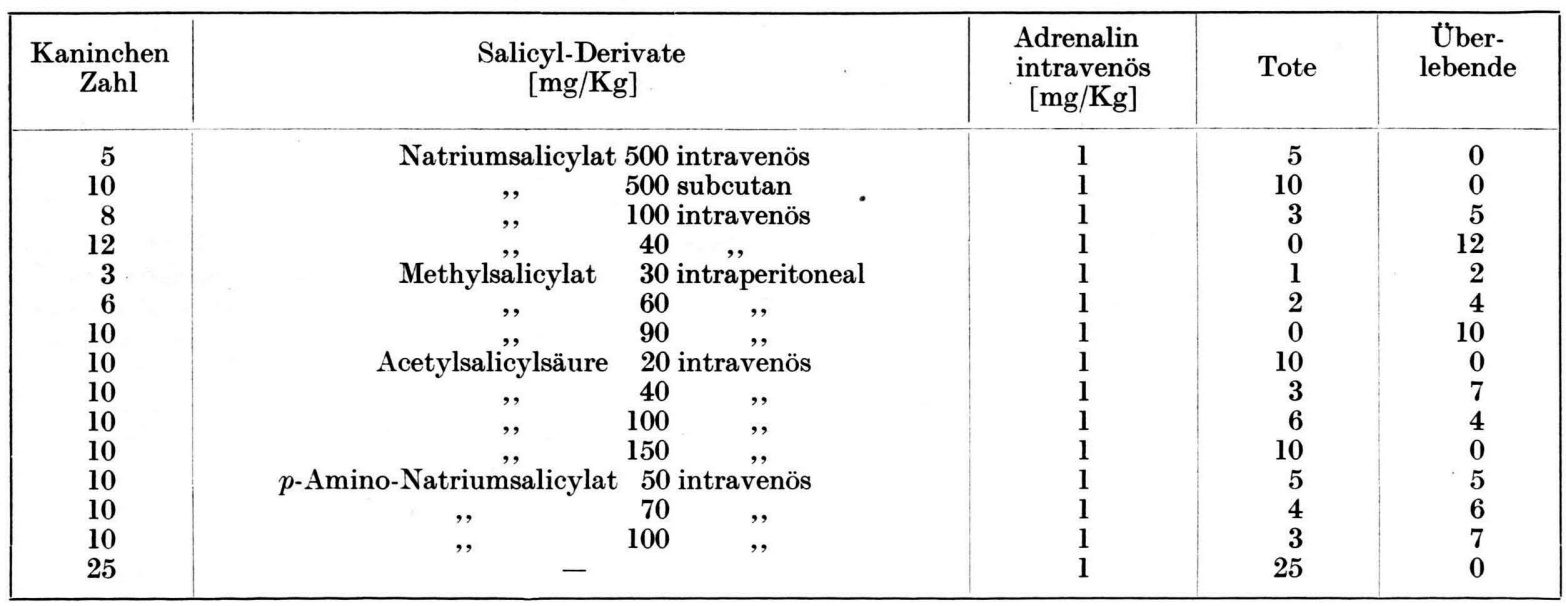

Tab. 1. Wirkung einiger Salicyl-Derivate auf das experimentelle Adrenalin-Lungenödem.

1 G. Lomeo u. P. De Bonis, Boll. Soc. ital. sperim. 31, 1437 [1955]. 